\title{
ALL CONFORMALLY FLAT PURE RADIATION METRICS.
}

S. Brian Edgar ${ }^{1}$ and Garry Ludwig ${ }^{2}$

\begin{abstract}
The complete class of conformally flat, pure radiation metrics is given, generalising the metric recently given by Wils.
\end{abstract}

\footnotetext{
1 Department of Mathematics, University of Linköping, Linköping, Sweden S581 83

2 Department of Mathematical Sciences, University of Alberta, Edmonton, Alberta, Canada T6G 2G1
} 
Wils [1] has recently given an example of a conformally flat metric representing pure radiation (i.e. energy-momentum tensor $T_{i j}=\Phi^{2} l_{i} l_{j}$, where $l_{i}$ is a null vector), and he points out, since this metric is not a plane wave, it contradicts Theorem 32.17 in Kramer et al. [2]. In the coordinate system $(u, v, x, y)$ this metric is given by

$$
g_{i j}=\left(\begin{array}{cccc}
-2 f(u) x\left(x^{2}+y^{2}\right)+v^{2} / x^{2} & -1 & 2 v / x & 0 \\
-1 & 0 & 0 & 0 \\
2 v / x & 0 & 1 & 0 \\
0 & 0 & 0 & 1
\end{array}\right)
$$

where $f(u)$ is an arbitrary function of $u$, which cannot be zero in curved spacetime.

Using a coordinate transformation $w=v / x$, Koutras and McIntosh [3] (see also [4]) have recently written this metric in a slightly different coordinate system $(u, w, x, y)$,

$$
g_{i j}=\left(\begin{array}{cccc}
-2 f(u) x\left(x^{2}+y^{2}\right)+w^{2} & -x & w & 0 \\
-x & 0 & 0 & 0 \\
w & 0 & 1 & 0 \\
0 & 0 & 0 & 1
\end{array}\right)
$$

They have also presented what appears to be a generalisation of Wils's metric, which is also a conformally flat metric representing pure radiation,

$$
g_{i j}=\left(\begin{array}{cccc}
-2 f(u)(a x+b)\left(x^{2}+y^{2}\right)+a^{2} w^{2} & -a x-b & a w & 0 \\
-a x-b & 0 & 0 & 0 \\
a w & 0 & 1 & 0 \\
0 & 0 & 0 & 1
\end{array}\right)
$$

where $a, b$ are parameters. This would seem to contradict Wils's claim that his example completes the class of conformally flat pure radiation metrics, which are not plane waves. Moreover, it is easy to confirm (eg. using Maple) that what appears to be an even more general metric,

$$
g_{i j}=\left(\begin{array}{cccc}
A(u, w, x, y) & -a(u) x-b(u) & a(u) w & 0 \\
-a(u) x-b(u) & 0 & 0 & 0 \\
a(u) w & 0 & 1 & 0 \\
0 & 0 & 0 & 1
\end{array}\right)
$$

where

$$
A(u, w, x, y)=-2 f(u)(a(u) x+b(u))\left(x^{2}+y^{2}+k(u) x+g(u) y+l(u) w+h(u)\right)+a^{2}(u) w^{2}
$$


and $a, b, f, g, k, l$ are arbitrary functions of $u$, is also a conformally flat metric representing pure radiation; but a little manipulation shows that, by a coordinate transformation of the type,

$$
w \rightarrow \alpha(u) w+\beta(u) x+\gamma(u), \quad u \rightarrow \lambda(u), \quad x \rightarrow x+\frac{b(u)}{a(u)}
$$

this metric can be transformed into the simpler form,

$$
g_{i j}=\left(\begin{array}{cccc}
-2 f(u) x\left(x^{2}+y^{2}+g(u) y+h(u)\right)+w^{2} & -x & w & 0 \\
-x & 0 & 0 & 0 \\
w & 0 & 1 & 0 \\
0 & 0 & 0 & 1
\end{array}\right)
$$

or, equivalently, in $u, v, x, y$ coordinates,

$$
g_{i j}=\left(\begin{array}{cccc}
-2 f(u) x\left(x^{2}+y^{2}+g(u) y+h(u)\right)+v^{2} / x^{2} & -1 & 2 v / x & 0 \\
-1 & 0 & 0 & 0 \\
2 v / x & 0 & 1 & 0 \\
0 & 0 & 0 & 1
\end{array}\right)
$$

where $f(u), g(u), h(u)$ are arbitrary functions of $u$.

We believe this metric (8) is a non-trivial generalisation of Wils conformally flat radiation metric, and indeed represents the whole class of conformally flat radiation metrics which are not plane waves. In curved space $f(u) \neq 0$, although $g(u), h(u)$ can be zero; clearly when both are zero (8) reduces to the Wils metric given by (1).

It is a little surprising that the metric (8) has been overlooked by Kramer et al. in Chapter $32,[2]$, since they seem to come close to giving it explicitly in their earlier Sections $27.5,6$, as we shall now show.

In Chapter 27 Kramer et al. [2] discuss the Kundt class [5] of spacetimes, i.e. spacetimes admitting a null congruence $l_{i}$ which is divergence-free $(\rho=0)$, and which therefore has a metric of the form

$$
d s^{2}=2 d \zeta d \bar{\zeta}-2 d u(d v+W d \zeta+\bar{W} d \bar{\zeta}+H d u)
$$

where we are using the notation of Chapter 27 in [2]. For the vacuum, pure radiation, or Einstein-Maxwell subclasses the Weyl tensor is algebraically special; pure radiation or null Einstein-Maxwell fields are aligned. 
Within the Kundt class, for vacuum, pure radiation, or null Einstein-Maxwell fields, when the Weyl tensor is specialised to either Petrov type III, $\mathrm{N}$ or $\mathrm{O}$, the field equations then lead to the two separate cases, [2]:

(i)

$$
W=W^{o}(\bar{\zeta}, u), \quad H=H^{o}(\zeta, \bar{\zeta}, u)+\left(W_{, \bar{\zeta}}+\bar{W}_{, \zeta}\right) v
$$

with the non-zero Weyl tensor components

$$
\Psi_{3}=\frac{1}{2} W_{, \bar{\zeta} \bar{\zeta}}, \quad \Psi_{4}=H_{, \bar{\zeta} \bar{\zeta}}
$$

It is shown in [2] that, for Petrov type $\mathrm{N}$ or $\mathrm{O}$, we are able to use the remaining coordinatetetrad freedom to put $W=0$. Therefore, we can conclude that the only solutions of Petrov type $\mathrm{O}$ are a subset of the plane-fronted waves, subject to the condition

$$
H_{, \bar{\zeta} \bar{\zeta}}^{o}=0
$$

(ii)

$$
W=W^{o}(\bar{\zeta}, u)-\frac{2 v}{\zeta+\bar{\zeta}}, \quad H=H^{o}(\zeta, \bar{\zeta}, u)+\frac{W^{o}+\bar{W}^{o}}{\zeta+\bar{\zeta}} v-\frac{v^{2}}{(\zeta+\bar{\zeta})^{2}}
$$

with the non-zero Weyl tensor components

$$
\Psi_{3}=\frac{\bar{W}_{\overline{,}}^{o}}{(\zeta+\bar{\zeta})}, \quad \Psi_{4}=(\zeta+\bar{\zeta})\left(\frac{H^{o}}{\zeta+\bar{\zeta}}\right)_{, \bar{\zeta} \bar{\zeta}}
$$

It is shown in [2] that, for Petrov type $\mathrm{N}$ or $\mathrm{O}$, we are able to use some of the remaining coordinate-tetrad freedom to put $W^{o}=0$. Therefore, we can conclude that the only solutions of Petrov type $\mathrm{O}$ must satisfy the condition

$$
\left(\frac{H^{o}}{\zeta+\bar{\zeta}}\right)_{, \bar{\zeta} \bar{\zeta}}=0
$$

We can easily integrate this to obtain

$$
H^{o}=f(u) x\left(x^{2}+y^{2}+2 \sqrt{2} \Re(Y(u)) x-2 \sqrt{2} \Im(Y(u)) y+h(u)\right)
$$


giving

$$
H=f(u) x\left(x^{2}+y^{2}+2 \sqrt{2} \Re(Y(u)) x-2 \sqrt{2} \Im(Y(u)) y+h(u)\right)-\frac{v^{2}}{2 x^{2}}
$$

where $Y$ is an arbitrary complex function of $u, h$ is an arbitrary real function of $u$, and we have substituted $\zeta=(x+i y) / \sqrt{2}$. The remaining coordinate freedom (see equation (27.11b) in [2]) can be used to put $\Re(Y(u))=0$, and the metric in (9) then becomes (8) under the substitution (17), with $g(u)=-2 \sqrt{2} \Im(Y(u))$.

Therefore the metric (8) represents all conformally flat pure radiation metrics in the Kundt class, which are not plane waves. Furthermore, it follows immediately from the Bianchi equations that all conformally flat pure radiation metrics are divergence-free, and thus fall into Kundt's class, [5]. So the class of metrics (8) - together with the class of plane waves given above by (9), subject to the substitution $W=0$ and $H=H^{o}$, where $H^{o}$ is obtained from (12) - completes the class of conformally flat metrics representing pure radiation.

For completeness, we add the following comments:

(1) Einstein-Maxwell null fields, which are a subset of the pure radiation fields obtained by applying Maxwell's equations, do not exist for the class of metrics (8); nor does this class permit massless scalar fields, nor neutrino fields.

(2) It is easy to deduce from the above discussion (and is implicit in Chapter 25, [2]) that the class of divergence-free pure radiation metrics whose Weyl tensor is of Petrov type $\mathrm{N}$ is also obtained from (9) in two separate cases as follows,

(i) $W=0$ and $H(u, \zeta, \bar{\zeta})$ satisfying $H_{, \bar{\zeta} \bar{\zeta}} \neq 0$. This is clearly a class of plane waves.

$$
g_{i j}=\left(\begin{array}{cccc}
-H^{o}(u, \zeta, \bar{\zeta})+\frac{v^{2}}{x^{2}} & -1 & \frac{2 v}{x} & 0 \\
-1 & 0 & 0 & 0 \\
\frac{2 v}{x} & 0 & 1 & 0 \\
0 & 0 & 0 & 1
\end{array}\right)
$$

and $H^{o}(u, \zeta, \bar{\zeta})$ satisfying $\left(\frac{H^{o}}{\zeta+\zeta}\right)_{, \bar{\zeta} \bar{\zeta}} \neq 0$. 
Once again, it follows immediately from the Bianchi equations that the Type $\mathrm{N}$ pure radiation metrics are divergence-free, and thus fall into Kundt's class. So the two metrics just given are the complete class of Petrov type $\mathrm{N}$ pure radiation metrics.

(3) The case of Petrov type III spacetimes has been discussed alongside Petrov II in Chapter 25, [2] and determined up to a degree of coordinate freedom. However, this is not necessarily the complete class of Petrov type III pure radiation metrics, but rather only the class of Petrov type III pure radiation metrics in the Kundt class, i.e. whose ray vector is divergence-free $(\rho=0)$; Petrov type III pure radiation metrics — unlike Petrov $\mathrm{O}$ and $\mathrm{N}$ - do not necessarily have a divergence-free ray vector.

Of course, there is the possibility that this new metric (8) is simply Wils' metric in a different coordinate system. However, we note in Wils' derivation — which was for a more general situation of a conformally Ricci flat type $\mathrm{N}$ pure radiation space — that he obtained a condition for $H^{o}$ (equation (3.8) in [1]) which is supposed to be the condition for conformally Ricci flat metrics. Clearly this condition should be satisfied automatically for conformally flat metrics; however, our solution (16) for $H^{o}$ does not satisfy this condition, in general. Therefore, since our expression for $H^{o}$ given by (16) leads to a conformally flat metric, but fails to satisfy equation (3.8) in [1] in general, Wils' condition (3.8) must be unnecessarily strong. Further, since Wils' metric in [1] satisfies (3.8) it must be less general than ours.

In addition, Skea [7] has used the invariant classification of the CLASSI program [8] to prove that the space-time (8) only reduces to the Wils case (1) when the functions $g(u)$ and $\mathrm{h}(\mathrm{u})$ are constants. The detailed classification by Skea of this metric, and of Wils metric [1], is available at the on-line exact solutions database in Brazil and in Canada, [7].

In a subsequent paper we shall present, via the GHP formalism, an alternative simple 
derivation of the metric found here and discuss in detail how to extend Wils' results.

\section{Acknowledgements.}

One author (BE) would like to thank the Department of Mathematical Sciences, University of Alberta for its hospitality while part of this work was being carried out, and for travel support from the Swedish Natural Science Research Council. The other author (GL) is grateful for the continuing financial support by the Natural Sciences and Engineering Research Council of Canada.

\section{References.}

1. Wils P. (1989) Class. Quantum Grav. 6, 1243.

2. Kramer D., Stephani H., MacCallum M. and Herlt E. (1980) Exact Solutions of Einstein's Equations (Cambridge: Cambridge University Press).

3. Koutras A. and McIntosh C. (1996) Class. Quantum Grav. 13, L47.

4. Koutras A. (1992) Class. Quantum Grav. 9, L143.

5. Kundt W. (1962) Proc. Roy. Soc. Lond. A 270, 328.

6. Geroch R., Held A., and Penrose R. (1973). J. Math. Phys., 14, 874.

7. Skea J. private communication, and at http://edradour.symbcomp.uerj.br and http://www.astro.queensu.ca/ jimsk.

8. Åman J.E. (1982). Manual for CLASSI: Classification program for geometries in General Relativity. Preprint, University of Stockholm. 